\title{
The optimal reconstruction size of nipple-areola complex following breast implant in breast cancer patients
}

Naomi Nagura-Inomata ${ }^{1,2^{*}}$, Yoshiko Iwahira ${ }^{2}$, Naoki Hayashi ${ }^{1}$, Takako Komiya ${ }^{2}$ and Osamu Takahashi ${ }^{3}$

\begin{abstract}
Background: Changes in the areola size after reconstruction of the nipple-areola complex (NAC) following mastectomy and breast reconstruction with a silicon implant in primary breast cancer patients have not been well examined. This study aimed to investigate time-dependent changes in the size of the donor and graft NACs and to assess clinical factors influencing these changes.

Methods: Fifty-eight consecutive patients who underwent nipple-areola reconstruction were retrospectively evaluated. Nipple-areola diameter was measured immediately after the NAC reconstruction and at each follow-up visit for at least 36 months.

Results: The donor NAC constituted $81 \%$ of the graft NAC at the time of operation. The size of the donor NAC gradually increased by up to $36.8 \%$ after the operation. The size of the graft NAC showed a decrease by $4.5 \%$ at 7 months, followed by recovery to the initial value. The ratio of the donor site size to the graft site size was increased at month 1 and then showed a gradual decrease to 1.08 at 36 months. A history of mastopexy or reduction for the donor site was independent factors associated with changes in the NAC size.
\end{abstract}

Conclusions: To achieve symmetry, the diameter of the donor NAC immediately after the reconstruction should be at least $20 \%$ smaller than that of the graft NAC, especially for patients without a history of additional operations.

Keywords: Nipple-areola reconstruction, Nipple-areola complex, Breast cancer, Skin graft

\section{Background}

Reconstruction of the nipple-areola complex (NAC) completes the final aesthetical step of breast reconstruction and restores the body image of breast cancer patients who have undergone mastectomy. An ideal reconstruction requires symmetry in position, size, shape, texture, and color, as well as permanent projection (Mohamed and Parodi 2011; Costa and Ferreira 2009; Nimboriboonporn and Chuthapisith 2014). NAC reconstruction is generally performed 2-3 months after the breast mound creation, as an out-patient procedure under local anesthesia. The major points of areola reconstruction are to recreate the pigmentation and texture

\footnotetext{
*Correspondence: naomi_inomata@hotmail.com

1 Department of Breast Surgical Oncology, St. Luke's International

Hospital, 9-1 Akashi-cho, Chuo-ku, Tokyo 104-8560, Japan

Full list of author information is available at the end of the article
}

typically associated with the opposite areola. Areola reconstruction, one of the popular procedures to achieve optimal cosmetic results, is accomplished by grafting from other sites, such as the contralateral areola or the upper inner thigh, or by intra-dermal tattooing (Farhadi et al. 2006; Bhatty and Berry 1997). Although tattooing is useful for areola reconstruction, special medical equipment, experience and periodical maintenance are required for optimize results. Therefore, grafting remains an important technique worldwide. According to Kargül et al. the best color match in NAC reconstruction was achieved by grafting from the contralateral areola rather than by grafting from the groin or tattooing (Kargül and Deutinger 2001). Although some studies have evaluated NAC reconstruction over the long term about the nipple projection (Few et al. 1999; Losken et al. 2001; Shestak et al. 2002; Banducci et al. 1999; Spear et al. 
2011), long-term evaluation of the size of the areola after grafting have not been examined. In this study, we first assessed the changes in the NAC size of both donor and graft sites in patients who had undergone nipple-areola reconstruction using a full-thickness skin graft from the contralateral areola following mastectomy with breast reconstruction. In addition, we also sought to identify clinical factors influencing the size of the NAC by comparing several parameters in different groups of patients.

\section{Methods}

\section{Patients}

This study included 58 consecutive primary breast cancer patients who had undergone NAC reconstruction using a full-thickness skin graft from the contralateral areola following mastectomy with breast reconstruction using a tissue expander/permanent implant at the Breast Surgery Clinic from March 2006 to December 2010 and had a minimum of 3 years of follow-up (Table 1). Patients who had undergone adjuvant irradiation were excluded. This study was approved by the ethics committee of the institutional review board of St. Luke's international hospital and permission was granted to access the patient's data. The need for written informed consent was waived because of the retrospective nature of the study.

\section{Reconstructive procedure}

Immediate or delayed two-stage breast reconstructions were performed using tissue expanders and silicone breast implants. Nipple-areola reconstruction was

Table 1 Patient data and demographics

\begin{tabular}{lll}
\hline & Median & (Range) \\
\hline Age (years) & 50.0 & $(32-67)$ \\
Volume of the implant (cc) & 310.0 & $(125-535)$ \\
\hline & $\boldsymbol{n}$ & $\%$ \\
\hline Total & 58 & \\
Age (years) & & \\
$\geq 50$ & 30 & 51.7 \\
$<50$ & 28 & 48.3 \\
Volume of implant (cc) & & 13.8 \\
$\geq 400$ & 8 & 86.2 \\
$<400$ & 50 & \\
Additional operations & & 48.3 \\
Mastopexy & 28 & 15.5 \\
Reduction & 9 & 5.2 \\
Augmentation & 3 & 31.0 \\
Timing of breast reconstruction & & 69.0 \\
Immediate & 18 & \\
Delayed & 40 & \\
\hline
\end{tabular}

performed about 3 months after the completion of the breast mound. We designed the new NAC area symmetrically of the opposite site. We marked to lower half of the nipple and outer rim of the areola at the donor site. The nipple was reconstructed using a composite graft taken from either the distal tip or the lower half of the contralateral nipple and the original position was closed directly (Fig. 1). The areola was reconstructed using a full-thickness skin graft from the outer rim of the contralateral areola, and the donor site was uniformly closed by suturing (Fig. 2). In this step, we carefully performed the suturing on the superficial fascial system, including the thick fiber bundle which located in the subcutaneous adipose tissue, to prevent the scar widening (Komiya et al. 2015). The NAC area at the graft site was de-epithelialized and the detached skin was grafted (Fig. 3). All reconstructive procedures were performed by a single plastic surgeon using the same technique. Pressure dressings were left in place for 14 days. After suture removal, the wound was supported by micropore skin tape $(3 \mathrm{M})$ at the donor site to fit the wound edge for up to 6 months.

\section{Nipple-areola size assessment and analysis of clinical factors}

Nipple-areola diameter was measured vertically and horizontally at the time of completion (intraoperatively) and at each subsequent follow-up visit at 1, 7, 12, 24, and 36 months postoperatively (Figs. 4, 5a). The average of the two diameters (Fig. 5b) and the ratio of donor and the graft site (Fig. 5c) was calculated. The following factors were assessed for associations with changes in the NAC size: age, volume of implant, a history of additional operations such as mastopexy or reduction surgery, and timing of breast reconstruction.

\section{Statistical analysis}

The $t$ test was used for comparison of continuous variables. The multivariate linear regression analysis was performed to evaluate relationships among the variables. A value of $\mathrm{p}<0.05$ was considered to indicate statistical significance. All statistical tests were performed using SPSS version 22.0 (SPSS Inc., Chicago, IL, USA).

\section{Results}

\section{Patients' characteristics}

The median age of the 58 patients was 50 years (range 32-67 years) (Table 1). The median volume of the permanent breast implant was $310.0 \mathrm{cc}$ (range 125-535 cc). Thirty $(51.7 \%)$ and 28 patients $(48.3 \%)$ were aged $\geq 50$ and $<50$ years, respectively. The volume of implant was $\geq 400 \mathrm{cc}$ and $<400 \mathrm{cc}$ in $8(13.8 \%)$ and in 50 patients (86.2\%), respectively. Twenty-eight patients (48.3\%) 

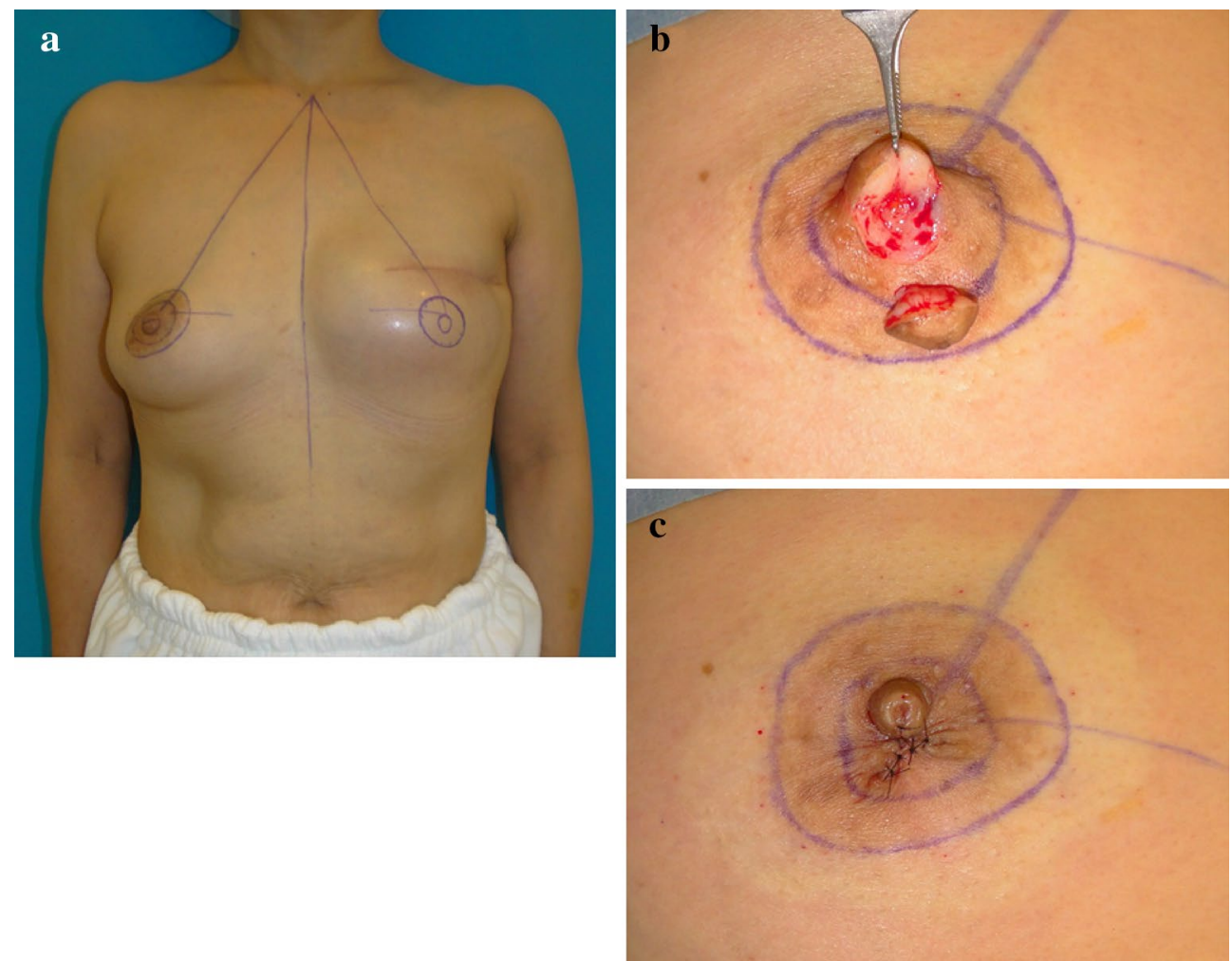

Fig. 1 The design of nipple-areola reconstruction performed by grafting from the opposite areola. We designed the new NAC area symmetrically of the donor site. Marking to lower half of the nipple and outer rim of the areola at the donor site (a). A composite graft was taken from the lower half of the nipple (b). The original position was closed directly (c) at the donor site

underwent mastopexy, 9 patients $(15.5 \%)$ had a reduction surgery, and the donor site breast was augmented in 3 patients $(5.2 \%)$ before the NAC reconstruction. The breast reconstruction was performed immediately following the mastectomy in 18 patients $(31.0 \%)$, and delayed reconstruction was used in 40 patients $(69.0 \%)$.

\section{Time-dependent changes in the NAC size}

The size of the donor NAC increased by up to $31.1 \%$ compared to the initial size at 1 month after the reconstruction and by up to $43.7 \%$ at 36 months in the horizontal direction. This change was larger than the change in the vertical direction (Fig. 5a). With regard to symmetry, the average diameter of the donor NAC constituted $81 \%$ of that of the graft NAC at the time of operation. The average diameter of the donor site gradually increased by up to $36.8 \%$ at 36 months compared to the initial value (Fig. 5b). In contrast, the size of the graft site decreased by $1.6 \%$ at 1 month in the horizontal direction and by $5.3 \%$ in the vertical direction (Fig. 5a). The average value gradually decreased by as much as $4.5 \%$ compared to the initial size at 7 months and then increased by up to $1.4 \%$ at 36 months (Fig. 5b). While the ratio of the donor NAC to the graft NAC at the operation was 0.81 , this ratio increased to 1.45 at 7 months and then decreased to 1.08 at 36 months (Fig. 5c).

\section{Factors associated with changes in NAC size}

As shown in Table 2, a history of additional operations was significantly associated with decrease in the size of the donor NAC $(p=0.002)$. The age and timing of the reconstruction did not influence the changes in the size of the donor site. With regard to the graft site, only a history of additional operations showed a trend towards influencing increase in the size of the corresponding NAC.

\section{Discussion}

To the best of our knowledge, this is the first study assessing long-term changes in the NAC diameter after reconstruction using a full-thickness skin graft from the contralateral areola following mastectomy with breast reconstruction. We focus on the areola size, not the loss of nipple projection. We showed that the size of the donor NAC gradually increased for 36 months, while the size of the graft site showed only slight changes 

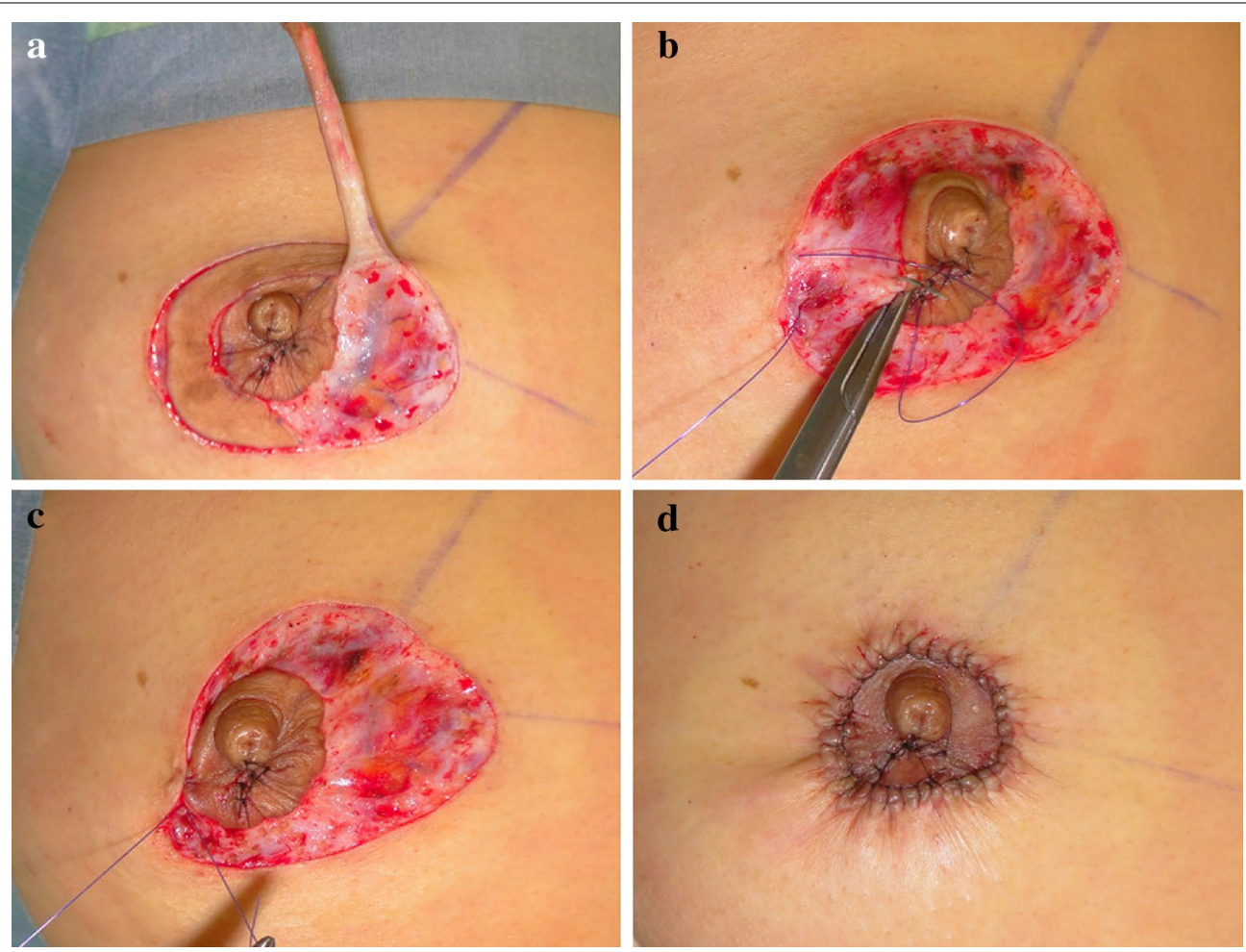

Fig. 2 A full thickness skin graft was taken (a). The original location of the NAC was closed directly by suturing the superficial fascia layer at the periareolar incision $(\mathbf{b}, \mathbf{c})$. The post-operative view $(\mathbf{d})$
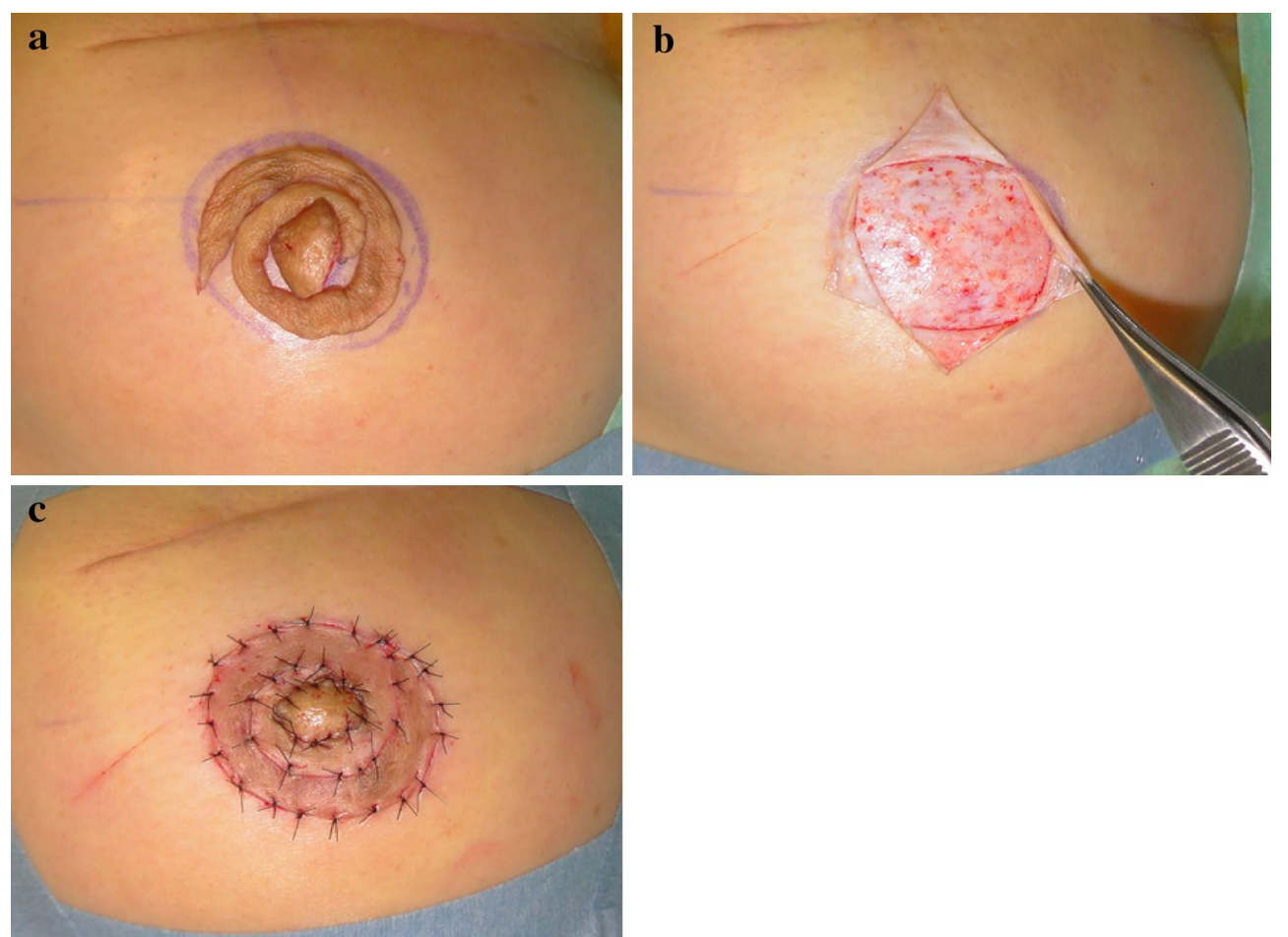

Fig. 3 The detached skin was repositioned (a) and the new NAC area was de-epithelialized at the graft site (b). The post-operative view (c) 

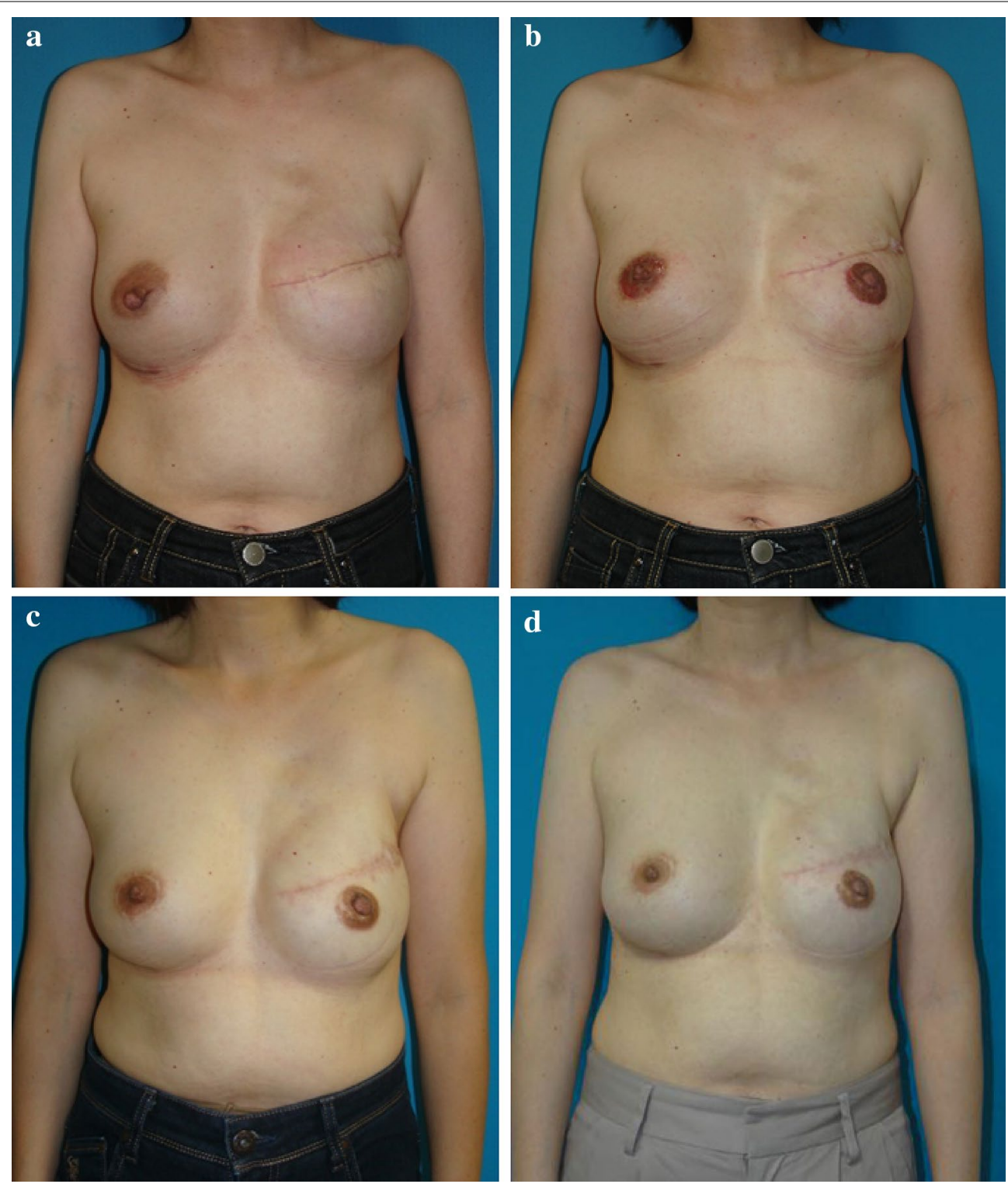

Fig. 4 A 47-year-old woman underwent mastectomy with immediate reconstruction with tissue expander. She exchanged the tissue expander for an implant 6 months later (a). Four months later, a left nipple-areola reconstruction was performed by grafting from the opposite areola (b). The picture shows 12 (c) and 36 months (d) after the nipple-areola reconstruction

within 24 months, followed by a relatively small increase. Despite constituting the smaller size of the donor NAC, it eventually became larger than the graft NAC, especially in patients who had large implant or without additional operations.

Areola reconstruction has been achieved by grafting as well as by tattooing. Although tattooing is a useful procedure and getting popular for areola reconstruction, special medical equipment is necessary and the technique requires training and experience to optimize results. Kargul et al. stated that the best color match on NAC reconstruction was achieved by grafting from the contralateral areola rather than by grafting from the groin and tattooing (Kargül and Deutinger 2001). Moreover, patients with large areola are, rather than reconstruct the areola large by tattooing, overlooking the areola reduction surgery at the same time as the areola reconstruction. Therefore, grafting still remains an important technique worldwide.

Postoperative complications such as hypertrophy, contraction, and graft failure have been described for fullthickness grafts (Stephenson et al. 2000; Leibovitch et al. 

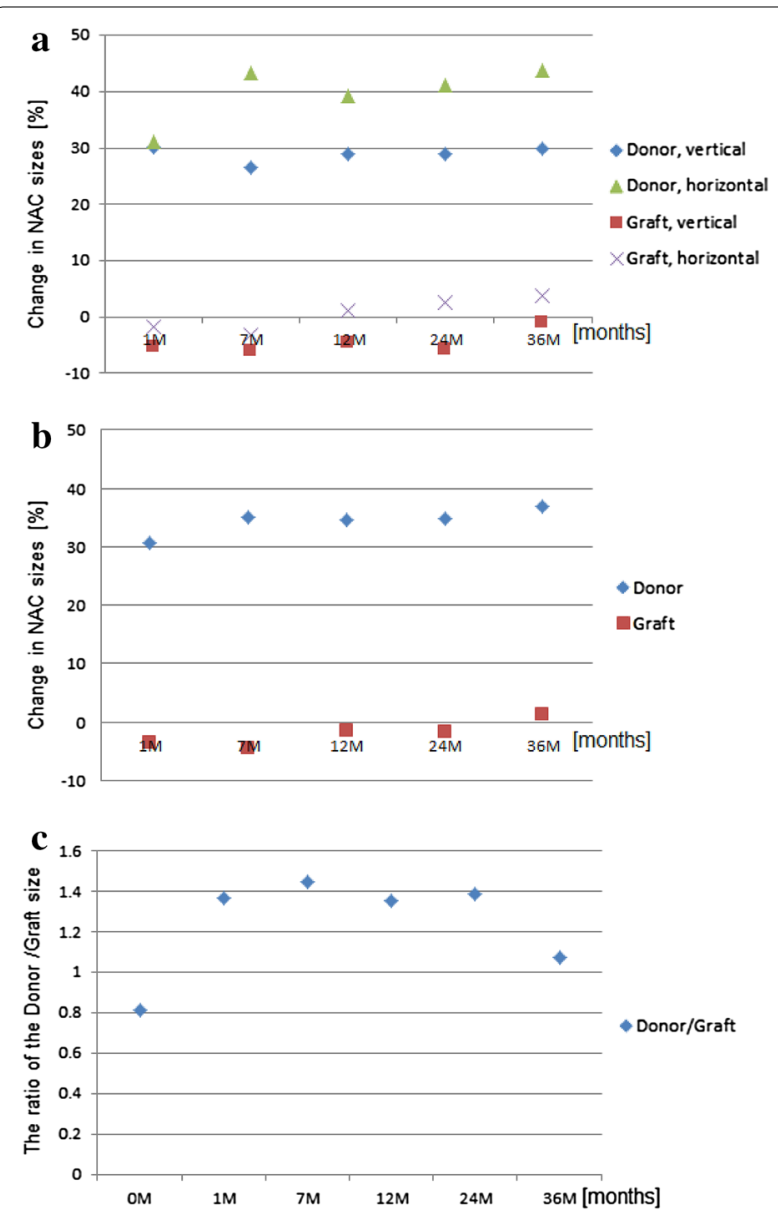

Fig. 5 Changes in nipple-areola complex (NAC) size after reconstruction using a composite nipple graft over time, (a) in vertical and horizontal directions, (b) in the average size of vertical and horizontal directions at the donor site and the graft site, and (c) the ratio of donor and the graft site. The initial NAC size was counted as zero and the percentage shows the ratio of size changes

2005). In general, however, full-thickness grafts tend to contract slightly with time, and, in this respect, our results are consistent with those of the previous studies. Interestingly, our study revealed an opposite trend for the size of the donor site. The size of the donor NAC showed and immediate increase reaching as much as $30 \%$ one month after the operation. Importantly, the periareolar edge was sutured for both the donor and graft sites when the areola was reconstructed using a full-thickness skin graft. Therefore, the differences in NAC size dynamics are unlikely to result from variations in the surgical technique. However, it is possible that the increase in the size of the donor NAC was due to expansion of the scar in the process of wound healing. The long-term use of the micropore skin tape after suture removal to prevent later stretching of the wound likely precluded such expansion at the graft site. The tendency of the donor NAC size to increase for at least 36 months postoperatively might be related to the softness of scar caused by maturing.

Mastopexy or reduction surgery were significantly associated with reduced size of the donor site. In this regard, these surgeries resulted in additional scars around the donor areola. Moreover, hypertrophic scars were removed at the time of the NAC reconstruction. This may have contributed to the contraction of the NAC. On the other hand, large implants had a tendency to increase the size of the donor NAC, which is a reasonable explanation of the expansion of the NAC as well as the breast skin. These factors might not have affected the size of the graft site because of the small magnitude of the changes, However, According to implant size, the number of patients who received large implant was too small to confirm the association with change in NAC size in this study. Further study is warranted for this point.

The usage of the purse-string suture technique for the periareolar skin closure has been reported to reduce the expansion of the areola and the loss of nipple projection (Weinfeld et al. 2008; Caterson et al. 2015). Bodin et al. suggested that removing a part of the contralateral nipple and areola might be the most effective technique in terms of stable long-term results (Bodin et al. 2008). Although we did not assess the efficacy of these published techniques in the present study, they may be useful to prevent the expansion of the donor NAC.

Our study has some limitations. First, this study had a retrospective design. Second, the use of the adjuvant hormone therapy or changes in the body weight, that may influence the size of the breast, were not assessed in this study because of the limited amount of data. This

Table 2 Statistical analysis of influence factors at $\mathbf{3 6}$ months

\begin{tabular}{|c|c|c|c|c|}
\hline & \multicolumn{2}{|c|}{ Change in size of donor sites } & \multicolumn{2}{|c|}{ Change in size of graft sites } \\
\hline & $\beta$ & $P$ value & $\beta$ & $P$ value \\
\hline Age (years) $(\geq 50$ vs. $<50)$ & 0.002 & 0.56 & -0.005 & 0.293 \\
\hline Volume of implant (cc) ( $\geq 400$ vs. $<400)$ & 0.001 & 0.073 & -0.0004 & 0.905 \\
\hline A history of mastopexy or reduction (yes vs. no) & -0.219 & 0.002 & 0.13 & 0.089 \\
\hline Timing of breast reconstruction (immediate vs. delayed) & 0.014 & 0.816 & -0.023 & 0.664 \\
\hline
\end{tabular}


warrants further study with a longer follow-up and larger sample size.

Our findings showed that the donor sites expanded about $36.8 \%$ while the graft sites didn't have a significant change. The results confirmed that the NAC size after reconstruction using a composite nipple graft was changed over time compared to the completion size. To account for this change, we should design the size of the areola at the graft site for NAC reconstruction as $20 \%$ larger than that of donor site, to prevent the asymmetry after few years. Furthermore, it is better to re-design the size of graft site intraoperatively when suturing the donor site was done.

The judgment of the degree to enlarge the graft site should examine the individual factors of the patient. The natural processes of contraction inherent with wound healing and aging cause the change in the size of areola in all NAC reconstructions. To successfully anticipate the long-term sizes of the donor and graft NACs, graft diameter and patient factors must all be considered. The donor site is usually enlarged, but the donor site in patient with a history of additional operations does not become larger than expected. In this case, we design the graft site larger than the opposite site, but it is smaller than when designing usually.

\section{Conclusions}

From our results, we suggest that the donor NAC should be planned at least $20 \%$ smaller than the graft NAC during the operation considering the time-dependent changes in the NAC sizes, especially in patients without a history of additional operations.

\section{Abbreviation}

NAC: nipple-areola complex.

\section{Authors' contributions}

I would like to add that all authors have directly participated in the planning, execution or analysis of the study; $\mathrm{NN}, \mathrm{Yl}$, and TK contributed to conception and design, and/or acquisition of data, and/or analysis and interpretation of data. NH conceived of the study, and participated in its design and coordination and helped to draft the manuscript. OT performed the statistical analysis. All authors participated in drafting the article or revising it critically for important intellectual content. All authors read and approved the final manuscript.

\section{Author details}

1 Department of Breast Surgical Oncology, St. Luke's International Hospital, 9-1 Akashi-cho, Chuo-ku, Tokyo 104-8560, Japan. ${ }^{2}$ Breast Surgery Clinic, YCC Takanawa Bild., 2,3/F Takanawa, Minato-ku, Tokyo 108-0074, Japan. ${ }^{3}$ Center for Clinical Epidemiology, St. Luke's Life Science Institute, 9-1 Akashi-cho, Chuo-ku, Tokyo 104-8560, Japan.

\section{Acknowledgements}

We would like to express our gratitude to Professor Ernest Manders, MD, from the Department of Plastic and Reconstructive Surgery, University of Pittsburgh Medical Center, Pittsburgh, PA, USA, for insightful comments and suggestions. We are also grateful to all the colleagues and staff at the Breast Surgery Clinic.

\section{Competing interests}

The authors declare that they have no competing interests.

Received: 12 January 2016 Accepted: 25 April 2016

Published online: 10 May 2016

\section{References}

Banducci DR, Le TK, Hughes KC (1999) Long-term follow-up of a modified Anton-Hartrampf nipple reconstruction. Ann Plast Surg 43:467-469

Bhatty MA, Berry RB (1997) Nipple-areola reconstruction by tattooing and nipple sharing. Br J Plast Surg 50:331-334

Bodin F, Bruant-Rodier C, Lutz JC, Himy S, Wilk A (2008) Reconstruction of the nipple-areolar complex: long-term results. Ann Chir Plast Esthet 53:334-341

Caterson SA, Singh M, Talbot SG, Eriksson E (2015) Reconstruction of areolar projection using a purse-string suture technique. Plast Reconstr Surg Glob Open 3:e453. doi:10.1097/GOX.0000000000000431

Costa MP, Ferreira MC (2009) Aesthetic quality of the nipple-areola complex in breast reconstruction with a new local graft technique. Aesthetic Plast Surg 33:774-779

Farhadi J, Maksvytyte GK, Schaefer DJ, Pierer G, Scheufler O (2006) Reconstruction of the nipple-areola complex: an update. J Plast Reconstr Aesthet Surg 59:40-53

Few JW, Marcus JR, Casas LA et al (1999) Long-term predictable nipple projection following reconstruction. Plast Reconstr Surg 104:1321-1324

Kargül G, Deutinger M (2001) Reconstruction of the breast areola complex. Comparison of different techniques. Handchir Mikrochir Plast Chir 33:133-137

Komiya T, Ito N, Imai R, Itoh M, Naito M, Matsubayashi J, Matsumura H (2015) Anatomy of the superficial layer of superficial fascia around the nipple-areola complex. Aesthetic Plast Surg 39:209-213. doi:10.1007/ s00266-015-0455-2

Leibovitch I, Huilgol SC, Hsuan JD, Selva D (2005) Incidence of host site complications in periocular full thickness skin grafts. Br J Ophthalmol 89:219-222

Losken A, Mackay GJ, Bostwick J 3rd (2001) Nipple reconstruction using the C-V flap technique: a long-term evaluation. Plast Reconstr Surg 108:361-369

Mohamed SA, Parodi PC (2011) A modified technique for nipple-areola complex reconstruction. Indian J Plast Surg 44:76-80

Nimboriboonporn A, Chuthapisith S (2014) Nipple-areola complex reconstruction. Gland Surg 3:35-42

Shestak KC, Gabriel A, Landecker A, Peters S, Shestak A, Kim J (2002) Assessment of long-term nipple projection: a comparison of three techniques. Plast Reconstr Surg 110:780-786

Spear SL, Schaffner AD, Jespersen MR, Goldstein JA (2011) Donor-site morbidity and patient satisfaction using a composite nipple graft for unilateral nipple reconstruction in the radiated and nonradiated breast. Plast Reconstr Surg 127:1437-1446

Stephenson AJ, Griffiths RW, La Hausse-Brown TP (2000) Patterns of contraction in human full thickness skin grafts. Br J Plast Surg 53:397-402

Weinfeld AB, Somia N, Codner MA (2008) Purse-string nipple areolar reconstruction. Ann Plast Surg 61:364-367 